\title{
Orthopaedic challenges for mucopolysaccharidoses
}

\author{
Andrea Borgo ${ }^{1}$, Andrea Cossio ${ }^{2}$, Denise Gallone ${ }^{2}$, Francesca Vittoria ${ }^{3}$ and Marco Carbone ${ }^{3 *}$
}

\begin{abstract}
Mucopolysaccharidoses (MPS) are a group of diseases characterized by abnormal accumulation of glycosaminoglycans (GAGs). Although there are differences among the various disease types, the osteoarticular system is always involved. The aim of the present study was to establish a framework for MPS-related orthopaedic manifestations and for their treatment. The authors, affiliated to three different Italian Orthopaedic Centres, report data taken from the literature reviewed in light of their accumulated professional experience. Bone alterations make up what is known as dysostosis multiplex, involving the trunk and limbs and with typical radiological findings. Joints are affected by pathological tissue infiltrations. The cervical spinal cord is involved, with stenosis and cervical and occipitocervical instability. In MPS there is a much higher incidence of scoliosis compared with healthy subjects without any particular distinctive feature. Kyphosis of the spine is more frequent and also more severe because of its possible neurological complications, and it is localized at the thoracolumbar level with a malformed vertebra at the top of the deformity. Evolving forms, and those associated with neurological damage, require anteroposterior spine fusion. The hip is invariably involved, with dysplasia affecting the femoral neck (coxa valga), the femoral epiphysis (loss of sphericity, osteonecrosis), and the femoral acetabulum which is flared. All these features explain the tendency to progressive hip migration. Genu valgum is often found (a deviation of the physiological axis with an obtuse angle opening laterally). This deformity is often localized at the proximal tibial metaphysis; it causes functional limitations and leads to an irregular erosion of the articular cartilage. In young patients who still have the growth plate, it is possible to execute a medial hemiepiphysiodesis, a temporary inhibition of cartilage growth, with progressive axis correction. In this paper, the characterisation of clinical features and the review of treatments are divided into separate sections based on the part of the body involved. The conclusions of each section are presented as a summary. One section discusses the high risk of anaesthesia-related complications requiring the collaboration of specifically trained personnel.
\end{abstract}

\section{Background}

Mucopolysaccharidoses (MPS) are a group of diseases characterized by abnormal accumulation of glycosaminoglycans (GAGs) in tissues. Although recent approaches, such as enzyme replacement therapy (ERT) and haematopoietic stem cell transplantation (HSCT), have partially improved the orthopaedic conditions, the osteoarticular system is invariably involved and thus the first specialist consulted is often the orthopaedic specialist.

Even though the pathogenesis of the disease is still not fully understood, it seems that GAG deposition in the connective tissue may play a key role in the osteoarticular manifestations through alterations in ossification and chronic inflammation.

\footnotetext{
* Correspondence: marco.carbone@burlo.trieste.it

${ }^{3}$ Institute for Maternal and Child Health IRCCS Burlo Garofolo, Via dell'Istria,

65, 34137 Trieste, Italy

Full list of author information is available at the end of the article
}

Some general features are shared by the different types of MPS, primary amongst which is joint stiffness due to deposition of GAGs during childhood with consequent thickening of capsules and ligaments. This is associated later on, as the disease progresses, with discrepancies in the deformed articular surfaces.

Stiffness is typically localized to the hands and shoulders, but it can affect all joints. This is a common feature of all MPS, except MPS IV where, on the contrary, there is joint hypermobility. Stiffness and articular deformity might require differential diagnosis with rheumatic diseases, but in MPS there are neither clinical nor humoral signs of inflammation [1].

The authors of the present paper are orthopaedic specialists with specific expertise in the field of paediatric pathology. The aim of the study was to review MPS-related orthopaedic manifestations, discussing concepts drawn

(C) The Author(s). 2018 Open Access This article is distributed under the terms of the Creative Commons Attribution 4.0 International License (http://creativecommons.org/licenses/by/4.0/), which permits unrestricted use, distribution, and 
from professional experience analysed in the light of recent scientific literature on the topic.

\section{General features: The dysostosis multiplex}

Radiologically, the skeletal deformities are labelled as dysostosis multiplex, characterized by severe abnormalities in the development of skeletal cartilage and bone. They consist of anomalies of the skull (macrocephaly, skull thinning), trunk (broadening of the clavicles, oar shaped ribs), spine (odontoid hypoplasia, thoracolumbar vertebral hypoplasia with angular kyphosis), limbs (short and wide diaphysis, irregular metaphysis, flattened epiphysis), hands (bullet-shaped phalanges), pelvis (flared iliac wings, flattened acetabulum, coxa valga, dysplastic femoral epiphysis), and knee (genu valgum) [2].

Short stature, which is a marker of inadequate growth, may be more or less pronounced [3]. In MPS I, IV, and VI, short stature is evident from infancy. In MPS II, stature can be higher, compared with average, up to the age of 8 years, after which growth velocity decreases progressively, finally resulting in short stature. In MPS III, stature is normal or close to normal. Furthermore, in MPS I, IV, and VI, the short stature is disharmonic, with a reduced trunk-to-limb ratio.

Because of impaired skeletal growth, delayed ossification and skeletal age are evident in radiological images.

In summary, in MPS patients the osteoarticular system is invariably involved with different degrees of severity depending on the type and stage of the disease.

\section{Spine: Kyphosis and scoliosis in MPS}

Spine involvement is common in the majority of MPS, although with great variability [4]. Flattened vertebral bodies (platyspondyly) are a typical feature of dysostosis. Wedge deformity of the vertebrae at the thoracolumbar junction (in MPS I, II, IV, and VI) can have dangerous consequences and shares some similarities with congenital kyphosis due to failure of formation. In fact, there exists a congenital defect in vertebral body formation at the thoracolumbar level that leads to progressive development of kyphosis in early infancy. The angular thoracolumbar kyphosis may progress, causing neurological damage as a result of the compression of the contents of the vertebral canal, with consequent ischaemic damage [2]. Standing up becomes more difficult because of forward misalignment of the trunk. Radiological evaluation of the vertebral deformity in anteroposterior and lateral views is performed with patients standing or sitting, measuring Cobb's angle (the angle between the upper and lower end vertebrae) for kyphotic and scoliotic (if present) assessment.

Magnetic resonance imaging (MRI) may help to identify preclinical signs of spinal cord involvement and localize them to the occipitocervical, cervical, or thoracolumbar regions. The role of somatosensory evoked potential
(SSEP) and motor evoked potential (MEP) registration in medullary compression diagnosis is, as yet, not fully established [5].

Orthopaedic treatment by means of bracing can improve stability and deambulation, but its influence on the evolution of deformities is not supported by clinical evidence. Once the deformity angle progresses beyond 40 degrees of amplitude it tends to aggravate, requiring a surgical approach [6], especially in MPS I and VI. Cases reported in the literature suggest that surgical treatment should be performed before adolescence, on average between the ages of 3 and 8 years $[2,7,8]$. Like all cases of kyphosis, whether congenital or in a skeletal dysplasia setting, angular kyphosis also requires a circumferential arthrodesis, which uses both an anterior and a posterior approach (Fig. 1). The literature suggests a posterior decompression associated with instrumentation and fusion in MPS IV [5]. Anterior fusion entails malformed vertebra removal and replacement with a bone graft from a tissue bank, preferably with the mechanical support of a mesh (there are different kinds of mesh with different forms and materials, mainly titanium and carbon). Posterior spine arthrodesis requires instrumentation (screws and metal bars) associated with bone grafts [7].

Despite the lack of consensus in the literature, in the case of mild deformities an isolated posterior vertebral arthrodesis can be performed to stabilise the surgical correction in association with periodic post-surgery controls [9].

A surgical approach to these deformities has a high risk of neurological complications that cannot always be predicted or avoided, even with modern electrophysiological monitoring techniques and with regular execution of preoperative MRI of the whole spinal cord. Considering that patients with MPS frequently have cervical and cervico-occipital malformations, in these cases it is not possible to use Halo traction, which is commonly employed in other paediatric pathologies. When the thoracolumbar kyphosis has progressive features, it is fundamental to opt for immediate surgery to avoid the development of major angular deformities which require longer operation times and more extensive corrections, thereby increasing the risk of neurological complications.

MPS IV patients often have kyphosis and cervicothoracic stenosis, which can cause neurological symptoms and require a surgical approach consisting of decompression and spinal fusion [10].

Scoliosis is not common in MPS and, when present, is usually associated with kyphosis and treated in conjunction with the management of sagittal plane deformities. Generally, scoliosis treatment is problematic because of trunk shortness and deformity in MPS, which makes it difficult to design a suitable orthopaedic brace. MPS III is the only type that presents different scenarios of spinal 


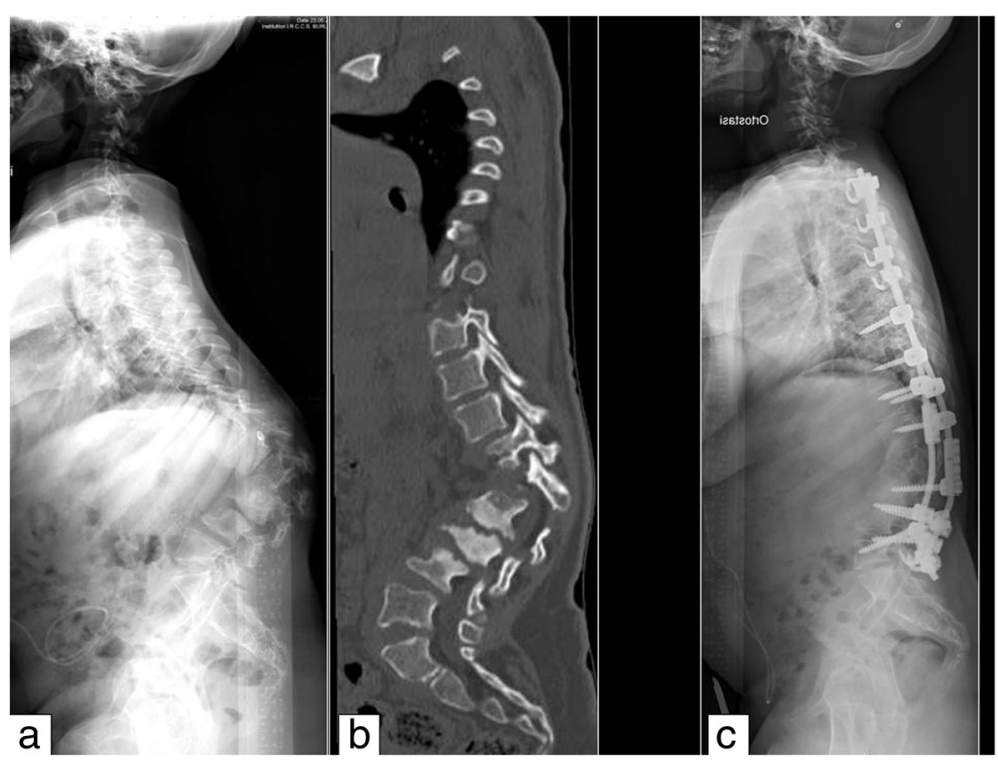

Fig. 1 Thoracolumbar kyphosis in a 12-year-old girl with MPS I. a Preoperative lateral x-ray showing severe wedge deformity of L1, with kyphosis of about $90^{\circ}$. b Computed tomography scan shows the dysostosis of the vertebrae. c Postoperative lateral x-ray showing good correction of the kyphosis after anterior fusion with tricortical bone graft and instrumented posterior fusion

involvement; the cervical spine is usually not affected and the costovertebral malformations typical of MPS are in a milder form. A wide-angle scoliosis may develop, and the surgical management must be tailored to the patient based on the prognosis.

In summary, spine involvement is common in MPS. Thoracolumbar kyphosis can cause neurological damage and may require early surgical treatment with anterior and posterior spine fusion.

\section{Upper limbs: Joint stiffness, trigger fingers, carpal tunnel syndrome}

Stiffness is the main feature of upper limb involvement in MPS and, in the worst cases, may develop during infancy, with a symmetric distribution and a progressive course. Shoulders are involved, with limited adduction. Elbows are limited in flexion-extension and especially pronosupination; this can be one of the first clinical signs of MPS, even in the milder forms. Hands are wide and thick, with ulnar deviation of the wrist. Metacarpophalangeal joints are fixed in extension, and interphalangeal joints (proximal and distal) are fixed in flexion, resulting in the so-called 'claw hand'.

The motion of the hand is limited by the infiltration of GAGs into the joint capsule, while the same kind of infiltration in the tendon sheath can cause stenosing tenosynovitis (trigger fingers). Trigger fingers at a paediatric age may be congenital when limited to the first finger; if other fingers are affected, an MPS diagnosis should be considered and examined in depth [11]. The treatment of trigger finger can be sometimes surgical, sometimes requiring not only flexor tendon pulley resection but also thorough cleaning of the tendon sheath with removal of GAG-infiltrated peritenonium.

Carpal tunnel syndrome is rare at a paediatric age, while it is present with higher prevalence in MPS I, II, and VI [11]. This manifestation is again linked to pathological tissue infiltration of the carpal tunnel, a narrow anatomical structure on the palm side of the wrist the walls of which are formed by carpal bones and transverse carpal ligament; the tunnel contains the flexor tendons of fingers and the median nerve [12]. Impairment of the latter can be assessed by clinical (thumb opposition impairment) and electromyographic examination, while the typical algic symptomatology is completely silent (burning pain in the first four fingers, especially felt at night). Surgical treatment of this syndrome, which is technically very easy, requires opening of the transverse ligament and removal of the pathological tissue from the tendon sheaths.

In summary, trigger fingers and carpal tunnel syndrome at a paediatric age must raise the suspicion of MPS.

\section{Hip involvement in MPS and possible indications for surgery}

Hip dysplasia is a common feature of MPS; it can cause gait impairment in children [13], leading to severe disability in young adults $[14,15]$.

MPS-related hip dysplasia varies depending on the type of MPS and can show different degrees of severity. The progression of hip dysplasia in children and adolescents with type I MPS-H (Hurler syndrome) after successful 
HSCT is well described in the literature with particular reference to poorly developed and vertical acetabulum, increased femoral neck-shaft angle, and gradual deformation of the femoral head. It has been suggested that these characteristics may contribute to hip instability with a consequent tendency to lateral and proximal migration. However, there is no consensus on the percentage and on the extent of the migration itself [14, 16-22]. Recently, a progressive increment of the anterior pelvic tilt has been reported [19], highlighting another important aspect of the anatomy and kinematics of the pelvis and the thoracolumbar spine in MPS I patients. Based on the progression of these anatomical alterations, a surgical reconstruction of the hip can be indicated in MPS I with a combination of both pelvic and femoral osteotomies to restore the normal anatomy of the hip [2, 16, 22, 23]. Hip reconstruction surgery performed in MPS I children can provide a stable, well-covered hip, but appears to be unable to prevent radiological deterioration and clinically significant hip arthritis in the long term. Thus, a total hip replacement may be needed in young adults [24].

Hip morphology and progression of hip alterations in MPS II are similar to that described for MPS I but to a milder extent. Similar to MPS I, there is no consensus regarding the incidence and extent of hip migration in MPS II [17, 18, 25]. Reviewing the literature on MPS I and II, it is clear that there is no strict correlation between the radiological parameters of hip dysplasia and the degree of pain and mobility impairment; some MPS patients may experience hip discomfort with a minor degree of radiographic dysplasia [18]. Furthermore, in MPS I patients there seems to be no correlation between radiographic parameters and clinical characteristics, such as age at the time of transplant (HSCT), ERT pre-HSCT, donor chimerism, and alpha-L-iduronidase activity post-HSCT [19].

Hip involvement in MPS III is less documented in the literature, but appears to be present in almost $50 \%$ of patients. Unlike the other forms of MPS, in MPS III wide deformities of the acetabulum are not common; the prevalent features are femoral head deformities and an increased femoral neck-shaft angle, which are rarely noticed before early adolescence [26, 27]. Therefore, MPS III patients may also experience hip pain. Generally, hip involvement in MPS III starts to be clinically relevant when the general conditions of these patients start to deteriorate. It is mostly treated with symptomatic therapy and steroid injections. Only rarely are major surgical treatments (femoral head removal, total hip arthroplasty) reported in the literature [23, 26, 27].

In MPS IVA, the hip shows anatomic alterations similar to MPS I but with a reduced tendency to lateral migration, especially during early infancy. In adolescents and in young adults, the main problems are the deformity of the femoral head and the poor development of the acetabulum $[28,29]$. Surgical hip management in infants with MPS IV is equivocal in the literature; some cases of hip reconstruction during infancy are reported, but there is a tendency to partial relapse of the migration. In adults, total hip arthroplasty should be considered [23].

In MPS VI, the progressive deformity of the femoral head as well as a lateral migration of the hip is already detectable in early infancy. Usually the migration is only partial and tends to be stable over time; pain and impairment are absent during the growing age. The literature on hip management in MPS VI during infancy is poor and the role of surgery is not clear while, in young adults, total hip replacement should be considered $[2,30]$.

In summary, hip dysplasia is a common finding in MPS. In MPS children, the hip should be followed-up with plain $\mathrm{x}$-rays. During the growing stage, surgical hip reconstruction can be considered, while in young adults an indication for total hip arthroplasty can be given. Indications for surgery are mostly based on the progression of the disease and on the symptoms.

\section{Lower limbs: What are the current indications for surgical treatment of genu valgum in MPS?}

In MPS patients a valgus deformity of the knee and ankle are often present, and a valgus deformity of the foot is also commonly observed, with the knee being the most frequently affected lower limb joint [31]. Valgus deformity is typically progressive from when ambulation starts and, in most cases, becomes severe enough to make surgery necessary. The deformity is mostly due to the proximal tibia, but the distal femur can also contribute. Progressive collapse and fragmentation of the tibial and femoral epiphysis becomes evident at radiographic examination [29].

The ankles of these patients are typically in valgus, with wedging of the distal tibial epiphysis and shortening of the fibula. In addition, valgus hindfoot can be detected, with some degree of equinus, and forefoot adduction, with some degree of supination and prominence of the fifth metatarsal head dorsally. In lateral $x$-rays, the tarsal bones appear small and irregular with plantarflexion of the talus.

Considering the progression of the deformity, close clinical follow-up is important to ensure timely intervention to prevent disability. Clinical evaluations should include assessment of lower limb alignment, range of motion (ROM) of the joints, and movement patterns. The knee may show a flexion contracture. In case of genu valgum, a good clinical method to assess deformity is measurement of the intermalleolar distance (in orthostatic and supine position). Of crucial importance is the evaluation of ligamentous laxity (with valgus varus stress tests) which can aggravate the deformity and is a distinctive element of MPS IV. 
Adequate clinical evaluation must be accompanied by the radiographic study of the pelvis and standing, full-length lower limbs in anteroposterior view to assess the mechanical axis measurements [32], and knee and ankle measurements (in particular the tibio-femoral angle, lateral distal femoral angle, and medial tibial proximal angle) [33]. Radiographic follow-up is essential in patients who show progression of the deformity at clinical evaluation.

In general, there are no definitive guidelines for the treatment of skeletal deformity in lower limbs in patients with MPS. For instance, the knee is considered pathological when the femoral-tibial angle is greater than $7^{\circ}$; however, the indication for surgical treatment should be given when it reaches $15^{\circ}$ of deformity [2, 34]. Regarding treatment options, hemiepiphysiodesis techniques (the so-called guided-growth techniques) have been shown to be simple and effective in reducing the deformity. However, since the growth potential is limited, correction is likely to take a long time. The guided-growth principle is applied through temporary load modulation of the growth of the physis. In the past this was achieved using Blount staples, nowadays with the so-called tension band plate $[34,35]$. It may be necessary to leave the devices in place for 2-3 years or more to obtain a good correction, but this does not seem to be associated with damage or closure of the physis (Fig. 2). Taking the characteristics of these patients into account, treatment should be started as soon as deformity becomes evident, even as early as 4 years old. Postoperative hospitalisation may be indicated depending on the complexity of the patient's condition; however, the procedure can usually be carried out as an outpatient $[29,35]$.

In cases of severe deformities associated with low growth potential, or in young adults with closure of the physis, osteotomy is recommended to obtain proper correction. Proximal tibial correction may be performed acutely through an oblique osteotomy, or gradually with an external fixator. For severe femoral valgus deformity, distal femoral valgus osteotomy and correction with an external fixator is the best option as it allows for ideal correction of the mechanical axis despite there being a high incidence of deformity recurrence after correction, mostly in the proximal tibia [36]. It is important to remember that osteotomies are major surgical interventions characterised by high complication rates and a long recovery time after surgery. In patients who are at high risk of losing the walking ability they had prior to surgery, these factors can lead to an impossibility of regaining preoperative function and, in some case, permanent loss of independent mobility [35]. The worsening of the deformity can lead to the development of symptomatic osteoarthritis requiring prosthetic replacement. There are only a few reports on this subject in the literature; however, they seem to indicate that these patients tend to require custom-made implants [37, 38].

Skeletal deformities are a characteristic feature of MPS and can be the cause of major disability. Those in the lower limbs often require repeated surgical interventions which should be carried out as early as possible, even at

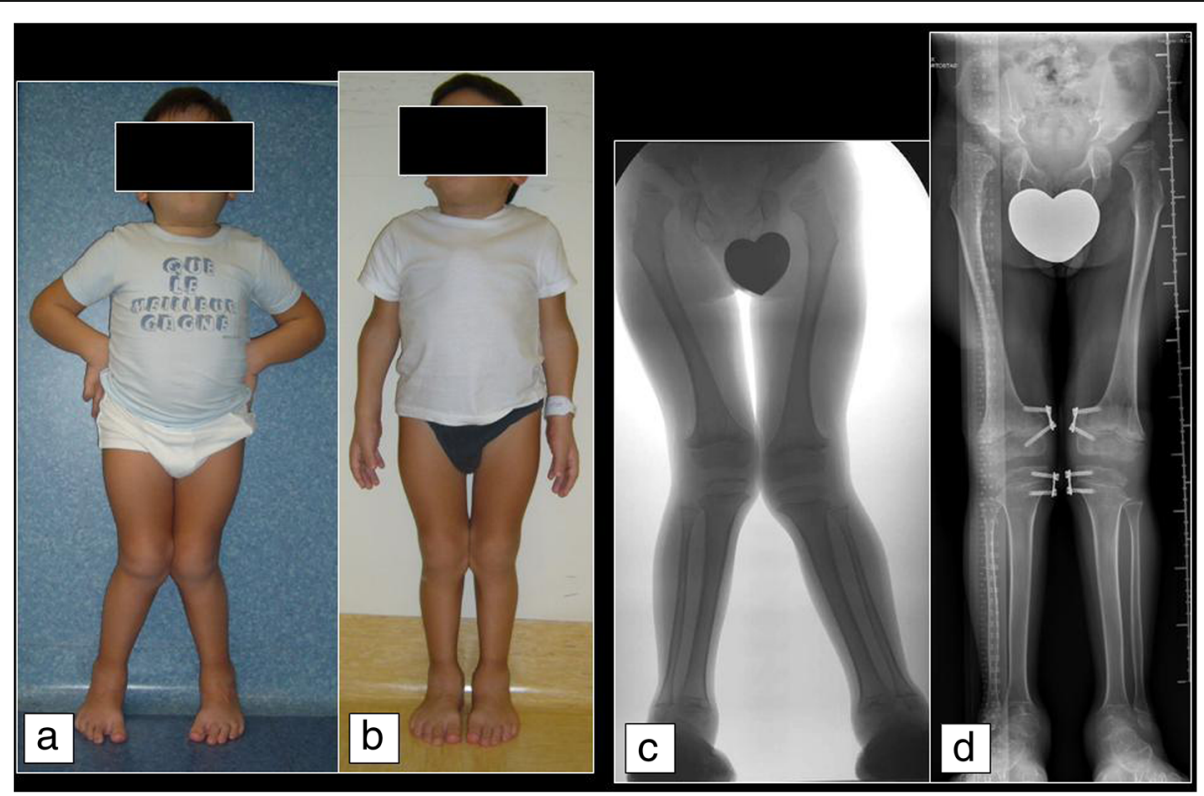

Fig. 2 Genu valgum in a 9-year-old boy with Morquio disease (MPS IVA). a Before temporary bilateral hemiepiphysiodesis of femur and tibia with Eight Plates. b After 2 years and 8 months, showing good correction of the valgus deformity. Clinical appearance of the patient $\mathbf{c}$ before surgery and $\mathbf{d}$ before plate removal 
the age of 4 years. In patients with major deformities, or those who are older, correction with hemiepiphysiodesis may be required even in the distal femur [35]. In these cases, it is important to attain some degree of overcorrection since there is a high percentage of recurrence of the deformity [21]. Treatment with guided growth procedures has proven to be effective in correcting lower limb defomities and increasing joint ROM by up to $30 \%$ [35].

Radiographic studies and gait analysis suggest that the deformities in the ankle and foot are primarily due to eversion of the subtalar and inactivity/weakness of the tibial posterior, both of which may be exacerbated by the valgus knee. In the majority of cases, foot and ankle problems can be treated with orthesis. In patients with good growth potential, the indication, also in the case of valgus ankle, is to perform a tibial hemiepiphysiodesis, while osteotomy is indicated for greater deformities in older patients, bearing in mind that, despite treatment, the deformity may recur [36].

In summary, temporary hemiepiphysiodesis with tension-band plates is an adequate solution for MPS patients with genu valgum, provided the growth potential is good.

\section{To remember: Complications during anaesthesia}

When giving indications for surgical intervention, the orthopaedic surgeon must take into due account the increased risks associated with general anaesthesia. This is especially true for patients affected by MPS as a result of reduced mouth opening, macroglossia, and frequent short and stiff cervical spine. The latter can be associated with cervical instability and stenosis (MPS IV and VI), which requires extra caution when performing intubation manoeuvres [2]. These patients should only be managed by anaesthesiologists who have experience in this field and with the aid of a radiological examination performed within the 12 months preceding the surgery. An emergency tracheotomy may become necessary at any moment [39].

In MPS II, GAG infiltration of the whole respiratory tract causes tracheal stenosis and may lead to severe complications that must be considered when general anaesthesia is planned [40].

In MPS I and IV, thoracolumbar kyphosis (possibly associated with cervicothoracic kyphosis in MPS IV) can cause neurological lesions with paraplegia; these may also occur in extraspinal surgical corrections, such as corrective osteotomy for hip dysplasia and osteotomy of the femur and tibia to correct knee valgus [41], even though the pathogenesis is not yet clear.

\section{Conclusions}

In summary, MPS patients are at high risk of neurological, anaesthesiological, and general surgical complications.

\section{Abbreviations}

ERT: Enzyme replacement therapy; GAG: Glycosaminoglycan; HSCT: Haematopoietic stem cell transplantation; MPS: Mucopolysaccharidosi(e)s; MRI: Magnetic resonance imaging; ROM: Range of motion

\section{Funding}

The publication costs for this paper in the IJP supplement were made possible with unconditional financial support from BioMarin, Sanofi Genzyme, and Shire. The sponsors had no input into the content of articles, which were independently prepared by the authors and have undergone the journal's standard peer-review process.

\section{About this supplement}

This article has been published as part of Italian Journal of Pediatrics, Volume 44 Supplement 2, 2018: Mucopolysaccharidoses: state of the art. The full contents of the supplement are available online at https://ijponline.biomedcentral.com/ articles/supplements/volume-44-supplement-2.

\section{Authors' contributions \\ Each author wrote a part of the manuscript. All authors have read and approved the final manuscript.}

Ethics approval and consent to participate

Not applicable.

\section{Consent for publication}

The patients or their parents/guardians gave written consent to the publication of their pictures.

\section{Competing interests}

The authors declare that they have no competing interests.

\section{Publisher's Note}

Springer Nature remains neutral with regard to jurisdictional claims in published maps and institutional affiliations.

\section{Author details \\ ${ }^{1}$ Orthopaedic Clinic, Padua University General Hospital, Padua, Italy. ${ }^{2}$ Department of Traumatology and Orthopaedic Surgery, San Gerardo Hospital Milano Bicocca Medical School, Monza, Italy. ${ }^{3}$ Institute for Maternal and Child Health IRCCS Burlo Garofolo, Via dell'Istria, 65, 34137 Trieste, Italy.}

Published: 16 November 2018

\section{References}

1. Aldenhoven M, Sakkers RJB, Boelens J, de Koning TJ, Wulffraat NM. Musculoskeletal manifestations of lysosomal storage disorders. Ann Rheum Dis. 2009;68(11):1659-65.

2. White KK. Orthopaedic aspects of mucopolysaccharidoses. Rheumatology (Oxford). 2011;50(Suppl 5):v26-33.

3. Morishita K, Petty RE. Musculoskeletal manifestations of mucopolysaccharidoses. Rheumatology (Oxford). 2011;50(Suppl 5):v19-25.

4. Peck SH, Casal ML, Malhotra NR, Ficicioglu C, Smith LJ. Pathogenesis and treatment of spine disease in the mucopolysaccharidoses. Mol Genet Metab. 2016;118(4):232-43.

5. Solanki GA, Martin KW, Theroux MC, Lampe C, White KK, Shediac R, et al. Spinal involvement in mucopolysaccharidosis IVA (Morquio-Brailsford or Morquio a syndrome): presentation, diagnosis and management. J Inherit Metab Dis. 2013;36(2):339-55.

6. Yasin MN, Sacho R, Oxborrow NJ, Wraith JE, Williamson JB, Siddique I. Thoracolumbar kyphosis in treated mucopolysaccharidosis I (hurler syndrome). Spine (Phila Pa 1976). 2014;39(5):381-7.

7. Garrido E, Tomé-Bermejo F, Adams Cl. Combined spinal arthrodesis with instrumentation for the management of progressive thoracolumbar kyphosis in children with mucopolysaccharidosis. Eur Spine J. 2014;23(12):2751-7.

8. Genevois KA, Garin C, Solla F, Guffon N, Kohler R. Surgical management of thoracolumbar kyphosis in mucopolysaccharidosis type 1 in a reference center. J Inherit Metab Dis. 2014;37(1):69-78.

9. Bekmez S, Demirkiran HG, Dede O, Ismayilov V, Yazici M. Surgical management of progressive thoracolumbar kyphosis in mucopolysaccharidosis: is a posterior-only approach safe and effective? J Pediatr Orthop. 2018;38(7):354-9. 
10. Baratela WAR, Bober MB, Thaker MM, Belthur MV, Oto M, Rogers KJ, Mackenzie WG. Cervicothoracic myelopathy in children with Morquio syndrome a: a report of 4 cases. J Pediatr Orthop. 2014;34(2):223-8.

11. Journeau P, Garin C, Polirsztok E, Jouve JL. Atteintes osseuses des mucopolysaccharidoses. Arch Pediatr. 2014;21(Suppl 1):S4-13.

12. Batdorf NJ, Cantwell SR, Moran SL. Idiopathic carpal tunnel syndrome in children and adolescents. J Hand Surg Am. 2015;40(4):773-7.

13. O'Brien A, Bompadre V, Hale S, White KK. Musculoskeletal function in patients with mucopolysaccharidosis using the pediatric outcomes data collection instrument. J Pediatr Orthop. 2014;34(6):650-4.

14. White KK, Harmatz P. Orthopedic management of mucopolysaccharide disease. J Pediatr Rehabil Med. 2010;3(1):47-56.

15. Mitchell J, Berger Kl, Borgo A, Braunlin EA, Burton BK, Ghotme KA, et al. Unique medical issues in adult patients with mucopolysaccharidoses. Eur J Intern Med. 2016:34:2-10.

16. Thawrani DP, Walker K, Polgreen LE, Tolar J, Orchard PJ. Hip dysplasia in patients with hurler syndrome (mucopolysaccharidosis type 1H). J Pediatr Orthop. 2013;33(6):635-43.

17. Stoop FJ, Kruyt MC, van der Linden MH, Sakkers RJB, van Hasselt PM, Castelein RM. Prevalence and development of orthopaedic symptoms in the Dutch hurler patient population after haematopoietic stem cell transplantation. JIMD Rep. 2013:9:17-29.

18. Ashby E, Baker M, Eastwood DM. Characterization of hip morphology in children with mucopolysaccharidosis types I and II. J Pediatr Orthop. 2016; 36(4):370-5.

19. J Langereis EJ, den Os MM, Breen C, Jones SA, Knaven OC, Mercer J, et al. Progression of hip dysplasia in mucopolysaccharidosis type I hurler after successful hematopoietic stem cell transplantation. Bone Joint Surg Am. 2016:98(5):386-95.

20. Breyer SR, Muschol N, Schmidt M, Rupprecht M, Babin K, Herrmann J, Stücker R. Hip morphology in MPS-1H patients: an MRI-based study. J Pediatr Orthop. 2016 [Epub ahead of print]. https://doi.org/10.1097/BPO. 0000000000000858.

21. Schmidt M, Breyer S, Löbel U, Yarar S, Stücker R, Ullrich K, et al. Musculoskeletal manifestations in mucopolysaccharidosis type I (hurler syndrome) following hematopoietic stem cell transplantation. Orphanet J Rare Dis. 2016;11(1):93.

22. Langereis EJ, Borgo A, Crushell E, Harmatz PR, van Hasselt PM, Jones SA, et al. Treatment of hip dysplasia in patients with mucopolysaccharidosis type I after hematopoietic stem cell transplantation: results of an international consensus procedure. Orphanet J Rare Dis. 2013;8:155.

23. Lewis JR, Gibson PH. Bilateral hip replacement in three patients with lysosomal storage disease: mucopolysaccharidosis type IV and mucolipidosis type III. J Bone Joint Surg Br. 2010;92(2:289-92.

24. Kennedy J, Noel J, O'Meara A, Mulhall K, Crushell E, Fogarty E, Kelly P. A long-term retrospective evaluation of functional and radiographic outcomes of pediatric hip surgery in hurler syndrome. J Pediatr Orthop. 2016;36(1):25-8.

25. Link B, de Camargo Pinto LL, Giugliani R, Wraith JE, Guffon N, Eich E, Beck M. Orthopedic manifestations in patients with mucopolysaccharidosis type II (hunter syndrome) enrolled in the hunter outcome survey. Orthop Rev (Pavia). 2010:e16:2-2. https://doi.org/10.4081/or.2010.e16.

26. White KK, Karol LA, White DR, Hale S. Musculoskeletal manifestations of Sanfilippo syndrome (mucopolysaccharidosis type III). J Pediatr Orthop. 2011;31(5):594-8.

27. de Ruijter J, Maas M, Janssen A, Wijburg FA. High prevalence of femoral head necrosis in mucopolysaccharidosis type III (Sanfilippo disease): a national, observational, cross-sectional study. Mol Genet Metab. 2013;109(1):49-53.

28. Borowski A, Thacker MM, Mackenzie WG, Littleton AG, Grissom L. The use of computed tomography to assess acetabular morphology in MorquioBrailsford syndrome. J Pediatr Orthop. 2007;27(8):893-7.

29. White KK, Jester A, Bache CE, Harmatz PR, Shediac R, Thacker MM, Mackenzie WG. Orthopedic management of the extremities in patients with Morquio a syndrome. J Child Orthop. 2014;8(4):295-304.

30. Dhawale AA, Thacker MM, Belthur MV, Rogers K, Bober MB, Mackenzie WG. The lower extremity in Morquio syndrome. J Pediatr Orthop. 2012;32(5):534-40.

31. Montaño AM, Tomatsu S, Gottesman GS, Smith M, Orii T. International Morquio a registry: clinical manifestation and natural course of Morquio a disease. J Inherit Metab Dis. 2007:30(2):165-74.

32. Stevens PM. Guided growth for angular correction: a preliminary series using a tension band plate. J Pediatr Orthop. 2007:27(3):253-9.
33. Paley D, Tetsworth K. Mechanical axis deviation of the lower limbs. Preoperative planning of multiapical frontal plane angular and bowing deformities of the femur and tibia Clin Orthop Relat Res. 1992;280:65-71.

34. Odunusi E, Peters C, Krivit W, Ogilvie J. Genu valgum deformity in hurler syndrome after hematopoietic stem cell transplantation: correction by surgical intervention. J Pediatr Orthop. 1999;19(2):270-4.

35. Cooper GA, Southorn T, Eastwood DM, Bache CE. Lower extremity deformity management in MPS IVA, Morquio-Brailsford syndrome: preliminary report of hemiepiphysiodesis correction of genu valgum. J Pediatr Orthop. 2016;36(4): 376-81.

36. Dhawale AA, Church C, Henley J, Holmes LJr, Thacker MM, Mackenzie WG et al. Gait pattern and lower extremity alignment in children with Morquio syndrome. J Pediatr Orthop B. 2013;22:59-62.

37. Atinga M, Hamer AJ. Total knee replacements in a patient with the Morquio syndrome. J Bone Joint Surg Br. 2008;90(12):1631-3.

38. de Waal Malefijt MC, van Kampen A, van Gemund JJ. Total knee arthroplasty in patients with inherited dwarfism - a report of five knee replacements in two patients with Morquio's disease type a and one with spondylo-epiphyseal dysplasia. Arch Orthop Trauma Surg. 2000;120(3-4):179-82.

39. Solanki GA, Alden TD, Burton BK, Giugliani R, Horovitz DDG, Jones SA, et al. A multinational, multidisciplinary consensus for the diagnosis and management of spinal cord compression among patients with mucopolysaccharidosis VI. Mol Genet Metab. 2012;107(1-2):15-24.

40. Gross ER, Lemmens HJM. Hunter syndrome in an adult: beware of tracheal stenosis. Anesth Analg. 2010;110(2):642-3.

41. Pauchard N, Garin C, Jouve JL, Lascombes P. Perioperative medullary complications in spinal and extra-spinal surgery in mucopolysaccharidosis: a case series of three patients. JIMD Reports. 2014;16:95-9.
Ready to submit your research? Choose BMC and benefit from:

- fast, convenient online submission

- thorough peer review by experienced researchers in your field

- rapid publication on acceptance

- support for research data, including large and complex data types

- gold Open Access which fosters wider collaboration and increased citations

- maximum visibility for your research: over $100 \mathrm{M}$ website views per year

At $\mathrm{BMC}$, research is always in progress.

Learn more biomedcentral.com/submissions 